\title{
Enseignement de la philosophie. Montaigne et la culture de l'alternance
}

\author{
Ensino de filosofia. \\ Montaigne e a cultura \\ da alternância
}

Teaching philosophy. Montaigne and the culture of alternation

\section{Résumé}

On peut s'intéresser de plusieurs façons à la question de l'enseignement de la philosophie. Je le ferai ici du point de vue de la pratique d'enseignement, c.-à-d. d'un point de vue didactique et/ ou pédagogique. Et cela, non pas en partant - ce qui serait tout à fait légitime - de la pratique d'enseignant de philosophie qui fut et est la mienne, mais plutôt en allant chercher chez certains philosophes de quoi instruire ma réflexion sur ces questions. Il y a en effet, chez les philosophes, et bien sûr, chez ceux qui se sont souciés, d'éducation, de très nombreuses analyses qui peuvent nous aider sur ce point. Certaines, comme celle de Montaigne, m'ont semblé particulièrement importantes, et je voudrais ici les exposer tout en rendant compte peu à peu des raisons pour lesquelles je les tiens pour importantes.

Mots-clés: Enseignement de la philosophie, didactique, Montaigne, philosophie de l'éducation.

\footnotetext{
* Departement des Sciences de l'Education. Université de Rouen. E-mail: hubert.vincent@univ-rouen.fr. 


\title{
Resumo
}

É possivel nos interessarmos de diversas maneiras pela questão do ensino de filosofia. Eu o farei aqui do ponto de vista da prática desse ensino, isto é, de um ponto de vista didático elou pedagógico. E farei isso não partindo - o que seria inteiramente legítimo - da prática de ensino de filosofia que foi e é a minha, mas, preferencialmente, buscando em certos filósofos como instruir minha reflexão sobre essas questões. Há nos filósofos - e com certeza naqueles que se ocuparam da educação - inúmeras análises capazes de nos ajudar nesse ponto. Alguns, como Montaigne, me pareceram particularmente importantes e gostaria de expô-los dando especial e detalhada conta das razões pelas quais eu os tenho por importantes.

Palavras-chave: Ensino de filosofia, didática, Montaigne.

\begin{abstract}
There are many ways to look at the question of teaching philosophy. I will do this here from the point of view of teaching practice, i. e. from a didactic and / or educational point of view. And that, not by leaving - which would be quite legitimate - of the practice of philosophy teacher who was and is mine, but rather by searching in some philosophers to instruct my reflection on these questions. There are indeed, among philosophers, and of course, among those who have cared, about education, about very many analyzes that can help us on this point. Some, like that of Montaigne, seemed to me particularly important, and I would like here to expose them while gradually accounting for the reasons why I consider them important.
\end{abstract}

Keywords: Teaching philosophy, didactics, Montaigne, philosophy of education. 


\section{I "Dévoluer"}

Je prendrai mon point de départ dans la notion de "dévolution", qui est comme on le sait un concept important de la didactique contemporaine et particulièrement mathématique. Qu'est-ce qui est en jeu avec ce terme peu habituel? Lorsqu'un théoricien invente un nouveau mot ou un nouvel usage d'un mot, cela peut correspondre à bien des motifs. L'un d'entre eux, me semble-t-il, correspond au souci de nouer dans ce terme ou usage des relations ou idées que le plus souvent nous mettons en opposition et attirer notre attention sur le fait que la réalité même s'est déjà chargée de ce lien. Peut-être bien que ces idées sont contradictoires, et peut-être que, dès lors ce qui en question sous ce terme est difficile à penser, mais au fond la réalité, ou certains aspects de cette réalité, nous mettent sur le chemin en indiquant que ce nouage se fait pourtant. C'est comme si cette réalité était toujours un peu plus riche et surprenante que ce que nous en pensions.

Je crois qu'il en va ainsi du terme de "dévolution" inventé par Guy Brousseau.

Ainsi la dévolution n'est-elle pas simplement transmission, sinon pourquoi avoir voulu ce nouveau mot? Elle n'est alors pas vraiment, ou pas seulement, transmission d'un contenu de connaissance ou d'un savoir. Elle est plutôt, de l'aveu même de l'auteur, transmission d'une responsabilité, ou encore dirons-nous, d'une initiative, ou d'un engagement. Mais comment peut-on transmettre une responsabilité ? Cela se transmet-il, une responsabilité, ou une initiative, ou un engagement? Si nous relions ces notions à celle de liberté, comment la liberté pourrait-elle être transmise? Il y a là semble-t-il une incompatibilité. L'initiative, l'engagement, c'est l'affaire du sujet et il n'y a que lui qui peut la ou le prendre.

Pourtant, l'idée que cette liberté se prend, plus ou moins, peu ou prou, et même en fonction de contextes particuliers, semble pouvoir être étudiée. Si la dévolution est bien transmission d'une responsabilité; et si c'est là quelque chose sur quoi nous avons peu de prise, ou même sur quoi nous ne devons pas chercher en avoir, il n'empêche que certaines situations, certaines relations, certains contextes y sont sans doute plus favorables que d'autres.

De plus, nous avons grandi, nous sommes, par certains aspects, venus à la suite d'autres, certains de nos actes comme certaines de nos pensées n'auraient pu venir hors d'un rapport de réception qui nous aura constitués. Nous avons des dettes en ce sens, et le savons, comme nous savons que, venus

1 La théorie des situations didactiques, Grenoble, ed. La pensée sauvage, 1995. 
après, nous avons repris ce qui nous aura été légué. Peut-être pas tout mais tout de même pas mal de choses. Il n'y a donc rien de surprenant dans le fait d'accorder que notre liberté est ainsi liée. Mais, de nouveau, ne peut-on pas demander dans quel cas et pourquoi un tel lien s'est fait, selon quelles conditions et contextes? Il y a peut-être des situations, des savoirs, des relations, dont on ne peut absolument rien faire ; d'autres en revanche qui se laissent reprendre, et qui nous auront permis nos propres choix. A quoi cela tient-il?

Qu'est-ce donc qu'une situation "dévoluante"? Un agir ou une attitude "dévoluante", si l'on peut parler ainsi ? Y faut-il une certaine parole, mais alors laquelle?

Compris ainsi, il m'a donc semblé que ce terme de dévolution n'était pas sans écho avec ce que je m'étais appris de la tradition philosophique de l'éducation, du moins une certaine tradition, et qu'il n'était pas non plus sans écho avec des questions qui semblent également plus contemporaines dans la discipline philosophique. Je remonterai du passé au présent selon deux ou trois étapes.

Une précision toutefois avant de commencer. Nous sommes des praticiens, nous nous intéressons aux pratiques. Et cela nous fait bien souvent suspecter des discours qui seraient un peu trop généraux, ou seulement théoriques comme on dit aujourd'hui, selon un usage curieux de ce terme. Comme praticiens et forts des théories dites pragmatiques, nous nous soucions avant tout du comment faire, et le plus souvent des règles qui nous permettraient d'assurer ce savoir-faire.

Mais je crois que sur ce point on peut avancer les deux choses suivantes.

D'abord que la théorie pragmatique ne peut être réduite au souci du comment et du bien faire ou encore au souci des règles qui nous conduiraient sûrement dans ce savoir-faire. Lécole pragmatique disait plutôt que si deux idées conduisent aux mêmes conséquences pratiques, alors il n'y a aucun sens à examiner longuement ces deux idées pour elles-mêmes. Limportant pour une idée, c'est qu'elle fasse quelque différence ou qu'elle ait quelque usage possible. Comme le soulignait Bouveresse, le pragmatisme, et particulièrement celui de Peirce, tient que "un concept, une proposition ou une théorie abstraite n'ont de sens déterminé que dans la mesure où nous pouvons en faire usage ou s'ils sont capables d'influencer notre conduite d'une manière spécifique et significative". ${ }^{2}$ La question n'est pas tant de faire jouer le critère d'utilité, mais celui "d'utilisabilité". ${ }^{3}$ C'est ainsi que je prendrai le terme.

2 Bouveresse,Jacques :Le mythe de l'intériorité.Paris: Ed. de Minuit, p. 541, 1987.

3 Idem. 
En second lieu, tout faire ne relève pas forcément d'un savoir-faire, et ne doit pas forcément en relever. Parfois nous agissons et répondons à la situation et, au moins assez souvent, certainement pas sans réflexion ou certainement pas sans ce que l'on pourrait nommer ruminations, attention, échauffement préalable et même précautions. Et certes, cela ne signifie pas que nous connaissions les règles de cette action. Souvent l'analyse de celles-ci vient après l'activité et du fait même de notre activité, "à sa suite", et alors nous cherchons à les élargir. Nous chercherons à dire plus loin toute l'importance de cette suite.

Alors donc à quoi conduit cette idée de dévolution, qu'est-ce qu'elle change, quelle autre et nouvelle réalité nous fait-elle voir, considérer, apprécier, et comment nous situe-t-elle par rapport à cette réalité ?

C'est sur Montaigne que je vais m'appuyer, dans cet article, pour cerner cette question, réservant à un deuxième article l'étude des prolongements plus contemporains des perspectives que je vais mettre au jour ici.

\section{II - Alternance}

Je prendrai appui, pour commencer, sur ce passage de son essai De l'institution des enfants. C'est bien un passage charnière car c'est vraiment à ce moment-là que Montaigne, après un moment d'échauffement où il s'est efforcé de remobiliser les éléments fondamentaux de son projet de pensée et d'écriture (exercer ou essayer son jugement), en vient à son objet présent, à savoir l'éducation et la pédagogie.

Que dit-il ?

On ne cesse de criailler à nos oreilles comme qui verserait dans un entonnoir, et notre charge ce n'est que de redire ce que l'on nous a dit. Je voudrais qu'il (le précepteur), corrigeât cette partie, et que, de belle arrivée, selon la portée de l'âme qu'il a en main, il commençait à la mettre sur la montre, lui faisant goûter les choses, les choisir, et discerner d'elle-même; quelque fois lui ouvrant chemin, quelque fois le lui laissant ouvrir. Je ne veux pas qu'il invente et parle seul, je veux qu'il écoute son disciple parler à son tour. "L'autorité de ceux qui enseignent nuisent à ceux qui veulent apprendre"(Cicéron). ${ }^{4}$ 


\section{Faire avec l'excès}

Au départ la mention d'une relation que, peu ou prou, nous reconnaissons tous, et encore aujourd'hui. Elle est faite de répétition, de criaillerie, de gavage, et chacun de ces termes mériterait d'être repris pour lui-même. Montaigne emploie le terme de criaillerie: ce n'est même pas cris, comme si à l'école il y avait une continuité d'un ton toujours au-dessus de l'ordinaire; non pas alternance entre des moments forts et faibles, des moments d'attention forte et faible, mais un continuum d'un ton ou d'une tension toujours la même. La voix qui transmet et la voix qui rappelle l'attention ne s'y distinguent également jamais, et il faut tout retenir également. Peu de place au jugement dès lors, pas de rythme, pas de trous ou de silences, pas d'interstices où quelque chose comme le jugement pourrait apparaître.

L'emploi par Montaigne du "notre charge" est aussi important, car le " notre » ici semble englober non seulement tous les élèves, qui doivent répéter la même chose et par suite ne pas se distinguer, mais aussi le maître, qui répète et redit un savoir qu'il aura lui-même entendu et répété. Dès ce point, on peut voir qu'il est fait peu de place au jugement, à la réflexion. Pas plus de jugement ici, mais bien exigence d'une répétition, toujours la même. Les professeurs enseignent ce qu'il faut savoir, ce qu'il convient à tous de savoir.

Ces termes dessinent le fond des analyses de Montaigne, - fond duquel il souhaite se détacher-, mais ils dessinent aussi notre fond commun dès lors que nous abordons cette question de l'école: nous reconnaissons là aussi l'école, nous qui lisons ces lignes, nous y reconnaissons tous quelque chose de l'école. Et ainsi et curieusement, ce fond semble être aussi le nôtre, et pas spécialement celui de l'époque de Montaigne.

Insister ainsi, comme je le fais, sur la relative permanence de ce fond a pour sens de nous mettre quelque peu en garde: il n'est peut-être pas quelque chose que l'on quitte aisément, au moins, et si chacun au travers des siècles, le retrouve, c'est peut-être qu'il est bien difficile d'y échapper et qu'il ne faut pas nous précipiter ou prétendre passer rapidement outre. Plus fondamentalement cela suggère que l'on ne doit pas forcément s'efforcer de quitter en tout point ce fond et qu'il a quelque droit.

Par exemple: "trop de connaissances" disons-nous, et alors"gavage". Mais que serait un souci de transmission de connaissances juste adaptées à notre mesure ou sans excès par rapport à une supposée mesure que nous serions ? Il faut bien qu'il y ait quelque excès de la connaissance pour que nous risquions d'excéder une mesure supposée. Cela bien sûr ne justifie pas toute pratique du gavage, mais du moins rend légitime l'expérience d'un certain 
excès, d'un "trop de connaissances", où nait précisément ce que nous visons lorsque nous parlons de gavage. Et peut-être faudrait-il avancer que le gavage résulte, non pas de l'excès comme tel, mais plutôt d'une méconnaissance de l'excès de certaine nourriture, comme si non seulement tout ce que nous donnions était forcément bon et adapté ou devait l'être, mais ne devait même pas donner lieu à une expérience de l'excès, de la distance à soi de la connaissance. Comme si autrement dit nous ne pouvions émettre des doutes, des interrogations, des questions, et d'abord des incompréhensions, sur ce que nous recevons, alors que cela même est façon de commencer à comprendre et faire sien. Douter, questionner, c'est garder en bouche, ni rejeter, ni avaler tout cru. En ce sens, on peut dire que l'expérience de l'excès, loin d'être opposé à l'apprentissage en est une condition. Nous savons bien, comme enseignant, que nous présupposons toujours un peu trop l'évidence ou l'allant de soi ou le devant aller de soi des connaissances que nous transmettons. Et nous savons bien combien il est difficile de se garder de ces dangers. Le gavage n'est donc pas l'excès, mais quasi son contraire: l'interdiction ou l'empêchement d'une expérience de l'excès.

Il y a également des raisons d'un autre type qui permettent de reconnaître cet excès. L'école est en effet lieu d'un cumul de connaissance. C'est là que l'on stocke les savoirs passés, que l'on souhaite transmettre et dont nous pensons qu'ils sont en mesure d'instruire ceux que nous éduquons. Pas d'école, semble-t-il, sans rapport à des connaissances accumulées dans le passé, et que l'on souhaite transmettre. Par là, et sans aucun doute, pas d'école sans une certaine forme d'écriture, et donc d'apprentissage de cette écriture d'abord. Elle est la condition, sous une forme ou une autre, de quelque chose comme une cumulation ou de stocks de connaissance. Dès lors, pas d'école sans décalage par rapport aux connaissances de l'expérience ou du présent. Et peut-être bien que parfois il faut un grand coup de balai ou un grand incendie pour nous débarrasser de ces stocks anciens qui nous pèsent, et peut-être que toute époque, - mais certaines plus que d'autres et la nôtre plus que d'autres- , ont eu ce goût d'un effacement de ce stock. Mais même dans une telle hypothèse, chaque époque n'a t-elle pas de nouveau refait des traditions, refait des stocks, et de même encore ne faut-il pas dire que les révolutionnaires ont aussi leur tradition, qui nous pèsent?

Il ne faudrait donc pas trop vite se précipiter et penser non seulement que l'on puisse mais que l'on doive rompre totalement, et d'un coup, avec cette forme de l'excès. Faire avec cet excès, est autre chose. Ce dans quoi s'engage Montaigne. 
Si ce fond est peu ou prou toujours là, - et on choisira de la nommer "forme scolaire 1"- on peut y faire rupture, contraste, et c'est bien ce à quoi Montaigne nous invite.

\section{Formes scolaires 1 et 2}

Il s'agit de renverser les choses, et changer de manière, d'emblée. La transmission, à l'école va dans un sens unique: elle prétend déverser le savoir dans l'élève. C'est ce rapport-là que Montaigne souhaite renverser, et ce renversement doit donner lieu à l'initiative de l'élève.

On pourrait penser que c'est ainsi bien simple: d'abord toute l'initiative à l'école et au maître, et puis initiative de l'enfant en sorte qu'après avoir tout donné à l'école, on en vient à tout donner à l'enfant. Il n'en est rien cependant et ce n'est pas la direction que prend Montaigne. Ce qui s'ouvre est plutôt et tout d'abord un partage de la relation: tantôt l'un, tantôt l'autre. Il y a maintenant, non pas un centre qui s'opposerait à l'autre, mais un espace et un temps fait d'une double initiative, ou d'une initiative partagée, en relation avec les objets, situations du monde, et les jugements prononcés par l'élève à ces occasions. "Tantôt... tantôt", tantôt l'initiative de l'élève, tantôt celle du professeur, l'une, peut-on supposer, enchainant sur l'autre.

Cela n'implique pas plus la fin de toute dissymétrie. Le précepteur en effet prend en charge l'espace lui-même de la relation; il n'est pas seulement un partenaire en relation, mais il veille aussi à l'espace ouvert ainsi, il veille à la qualité d'un espace où quelque chose comme des initiatives de l'enfant, mais aussi des initiatives de l'enseignant soient possible, ainsi qu'à leur enchaînement l'une sur l'autre. Il veille au rapport lui-même. Il est dans la relation, et il est en dehors de cette relation pour la prendre en garde."Tantôt lui ouvrant le chemin, tantôt le lui laissant ouvrir", comme dit Montaigne, et c'est bien le précepteur qui veille ainsi à ce tempo. Tout se passe ainsi comme si le souci de l'initiative en contexte scolaire n'avait de sens que dans le cadre non seulement d'une initiative partagée, mais de quelqu'un qui veille et accompagne ces initiatives. Peut-être les laisser aller, autant que les reprendre, comme nous le verrons plus loin. On nommera cette forme: "forme scolaire 2", pour cette raison, qu'en dépit de sa différence d'avec la première, elle est bien quelque chose de l'école: un maître qui veille aux initiatives les reprend, - et les corrige par exemple - , autant qu'il relance tant sa propre activité que celle de l'enfant, autant qu'il est ouvert à d'autres occasions de jugement. 
En se rappelant la façon dont Socrate discutait, - que Montaigne a bien en tête lorsqu'il écrit ces pages- le précepteur est attentif non seulement à l'échange lui-même, dans son contenu, qu'à sa forme. Il cherche, entre autres, à avoir une mémoire de ces échanges, et il peut par exemple remettre en mémoire ce qui a été dit, faire le point sur tel résultat de la discussion ou du travail, comme il peut faire des haltes, chercher à recueillir ce qui a été dit, pour repartir ensuite. Il garantit ainsi la continuité d'un travail ou d'une réflexion.

Si une certaine assymétrie demeure, on peut penser qu'elle sera peu à peu dépassée ou que l'élève en viendra lui aussi à prendre en charge la continuité même. Par exemple, à se souvenir de ce qui a été dit pour le comparer à ce qui est dit maintenant, et ainsi entrer dans le souci d'une continuité de la réflexion comme du travail. C'est sans doute là une réponse à la question de savoir de quel type de responsabilité on parle lorsque l'on parle de transfert de responsabilité en contexte scolaire: transférer pas seulement une mémoire, mais bien la capacité d'une mémoire et d'une réflexion, un faire mémoire. Lélève revient, de lui-même, à ce qui aura été dit, à ce qu'il aura dit, le reprend. Il pense.

Quoi qu'il en soit de ce dernier point, la forme, ou le cadre, ou le rituel dans lequel Montaigne veut situer l'institution des enfants, n'est pas autre chose que cette initiative partagée. Forme scolaire 2, disions-nous.

\section{Une attention double : l'éleveur et le chasseur}

Qu'est-ce que cela change, demandais-je, plus haut, qu'est-ce que cette idée change? Comme on l'a vu, elle change pas mal de choses, déjà. Mais je voudrais mettre l'accent sur le point suivant. Ce qui change c'est, me semble-t-il, l'attention de l'enseignant: son attention devient attentive, pourrait-on dire, c.-à-d. qu'elle nait proprement. Attentive, d'une part à ce qui vient de l'élève, ainsi qu'à son propre pouvoir d'enchaîner sur cette initiative. Attentive aussi à ce que lui-même propose, mais précisément, en tant que cela a ou non de l'effet chez celui qu'il éduque, en tant que cela peut être repris ou non par l'élève. Non pas: "je te donne le savoir et tu dois le répéter, et moi je dois m'assurer que tu l'as bien compris" mais "je te donne quelque chose et suis attentif à ce que tu en fais, aux transformations que tu lui fais subir". Auparavant, dans le modèle initial rejeté par Montaigne, il n'y a pas d'attention à proprement parler: mais seulement la répétition et la vérification de ce que chacun doit savoir. Ce qui, certes, n'est pas rien. Mais pas d'attention, au sens où l'attention est quelque chose qui s'éveille ou qui attend; je veux dire au 
sens où l'attention est attention à quelque chose qui vient, ou ne vient pas, qui peut venir ou ne pas venir, qui est en un sens attendu, mais pas toujours là. Le chasseur en ce sens est attentif; il ne sait quand, comment, ni même où l'animal apparaîtra, mais il pense que cet apparaître aura lieu ou du moins l'attend.Il pense qu'il peut être surpris. Qu'est-ce qu'une attention éveillée, sinon une attention qui guette. Et un enseignant pourrait-il être attentif, s'il n'avait dans l'idée par laquelle les voies seront quelque peu surprenantes, ou du moins peuvent l'être? Bruner, dans son texte classique sur l'étayage mentionne, à la fin de son texte,- et donc un peu en marge des lignes d'analyses majeures de son article- que ce qui fait la différence entre un enseignant et une machine à apprendre c'est que le premier sait reconnaître que parfois il est devant des élèves qui emploient des stratégies vraiment bizarres, et semblent vraiment savoir ce qu'ils font tout en entrant dans des chemins improbables. Ils doivent se faire alors, et se font le plus souvent, herméneutes. Parfois donc, il peut y avoir surprise, et attention en ce sens. ${ }^{5}$.

Mais l'enseignant est-il lui-même un chasseur et doit-il être attentif en ce sens? Ce n'est pas sûr. N'est-il pas plutôt un éleveur, nettement plus attentif aux régularités, aux répétitions, aux attentes bien connues et régulières ? Ou encore à ce qui "ne pourra manquer de venir?". Ce que nous disions plus haut de l'école comme lieu d'une certaine cumulation et de la transmission des savoirs passés recueillis, va dans le même sens. L'école est culture, elle est liée à l'agriculture, à l'élevage, aux sédentaires.

On peut dès lors avancer que l'attention de l'enseignant doit être double ou même qu'elle est double. Pour une très large part, elle est conduite par une attente dont on sait déjà le but, et au sujet de laquelle il n'a pas vraiment d'inquiétude. Mais pour une autre part, ne faut-il pas, au moins parfois, une attention à l'inattendu, à ce qui sort des sentiers battus, à ce qui surprend. Etre seulement un éleveur conduirait à ne rien vouloir savoir des inattendus et à s'assoupir. Mais être exclusivement un chasseur manquerait à reprendre les voies, bien connues, de tout apprentissage.

Ce qui est en question, c'est ainsi quelque chose comme la naissance d'une attention, et la question de savoir quelle est la forme d'attention de l'enseignant. Par hypothèse, nous dirons qu'elle est double. Accueillir des initiatives, c'est certainement, les "ramener" vers des chemins plus simples, avec tout ce que ce terme suppose de patience et d'attente, comme on ramène petit à petit des bêtes dans leur enclos. Et le pâtre qui "ramène" ses bêtes est heureux de 
les ramener ainsi: elles se sont dispersées relativement dans le champ, bien sagement, et voilà maintenant que sonne l'heure du rassemblement. Mais c'est aussi, à la façon du chasseur, être attentif à des initiatives étonnantes, des surprises, des écarts pas vraiment anticipés, des voies bizarres et non calculées, non prévues. "Tantôt, ... tantôt", de nouveau, sans que l'on puisse le savoir à l'avance. Nous chercherons à confirmer cette hypothèse dans la suite de nos analyses. On voit qu'elle articule les deux formes scolaires 1 et 2 .

\section{III - Conditions et modalités de l'alternance.}

En suivant maintenant les situations que Montaigne mentionne, que peut-on dire de ce qui se passe là, de ce qui advient là, dans la forme d'attention ainsi ouverte? Je prendrai quatre de ces situations.

\section{Mettre en exemples}

Montaigne écrit ceci :

Que, ce qu'il viendra d'apprendre il le lui fasse mettre en cents visages et accomoder à autant de divers sujets, pour voir s'il l'a encore bien pris et bien fait sien. ${ }^{6}$

La dévolution ici concerne donc les exemples, et le travail ou l'invention d'exemples. Je ne m'y arrêterai pas trop longtemps, car cela peut sembler aller de soi et être trop évident. Il n'en est rien, me semble-t-il. Tout d'abord parce qu'il n'est pas du tout certain que cela soit fait habituellement, loin de là. Ensuite parce qu'il y a bien, dans le fait que l'on demande aux élèves ou étudiants un nouvel exemple, ou de trouver eux-mêmes des exemples, un risque, et, ce faisant, le début d'une prise de responsabilité: c'est à eux de juger, c'est à eux de reprendre l'idée, en pensant l'avoir comprise, puisqu'on leur demande un exemple et de proposer quelques nouvelles illustrations. Et bien souvent dans ce cas, les élèves ne font que donner des exemples très proches de ceux qu'ils ont déjà vus. Ils osent peu sortir du déjà vu et du supposé attendu que le cours antérieur a déjà dessiné. Ils songent rarement à chercher ailleurs les 
exemples autres, dans leur vie certes, mais aussi et simplement dans ce que leur suggère leur première compréhension et ce qu'elle leur permet de penser, leur permettrait peut-être bien de penser. Pourquoi pas cela aussi; pourquoi ne pourrait-on pas dire cela aussi ? Il n'y a nulle facilité à sortir du supposé bien connu, ou attendu.

Et là, on peut avancer la chose suivante: on peut prendre appui sur "l'attendu", sur la forme scolaire 1. Je veux dire que les exemples un peu inattendus auront d'autant plus de force qu'ils supposent la différence avec ce qui était attendu et que les élèves peuvent la reconnaître (et le maître aussi peut-on espérer). Au fond, il n'y a pas qu'à l'école que notre esprit comme notre action fonctionnent selon une certaine attente: le monde social est fait d'une telle attente. En sorte que le jeu propre de l'école, sa force propre, est que dans ce lieu-là les écarts sont plus surprenants autant que plus accueillis. Bien sûr, nous reviendrons plus loin à cette thèse.

Et je crois que, là encore, nous retrouvons la double attention dont nous parlions plus haut: certains exemples seraient manifestement en dehors, d'autres, un peu neufs et inattendus, outre tous ceux qui resteraient, au moins au début, dans la conformité avec l'attendu ou supposé tel. Parfois donc ramener, parfois s'ouvrir.

Comme nous retrouverions la remarque précédente au sujet de la dévolution du travail de ces exemples aux élèves eux-mêmes : il suffit que le travail des exemples, au sens dit, et selon cette double attention, devienne un peu régulier, pour que les élèves s'y impliquent et s'y engagent. Ils savent que là il peut y avoir enjeux, et risques.

\section{Le trotter. Questions de déontologie ou de vertus}

Il est bon qu'il le fasse trotter devant lui pour juger de son train, et juger jusqu'à quel point il se doit ravaler pour s'accommoder à sa force. A faute de cette proportion nous gâtons tout; et de la savoir choisir, et s'y conduire bien mesurément, c'est l'une des plus ardues besognes que je sache; et est l'effet d'une haute âme et bien forte, savoir condescendre à ses allures puériles et les guider. Je marche plus sûr et plus ferme à mont qu'à val. ${ }^{7}$ 
Ce qui advient dans l'espace ainsi ouvert c'est le trotter, c.-à-d. une allure maladroite, celle de l'enfant bien sûr, qui, comme on dit, tatônne, avance timidement, et de façon peu ou prou chaotique. C'est sans doute la première caractéristique de son initiative : un certain chaos de l'activité, certaines maladresses, des chemins bizarres, etc. Or cela est tout fait central pour Montaigne, car cela fixe selon lui au moins un élément de la déontologie et plus encore de la valeur de l'enseignant. Il faut, dit-il, en effet "une haute âme et bien forte pour se ravaler à ces allures puériles". Il n'explicite pas trop ou ne justifie pas cette affirmation. Peut-être est-elle trop évidente pour lui. On peut cependant tenter de le faire.

Qu'est-ce que c'est qu'une haute et une forte âme? Haute ici dit la chose suivante: oublier, ou mettre de côté mes propres fins, pour m'intéresser à ce que fait l'enfant; peut-être bien ai-je, comme enseignant, comme éducateur, des fins, des buts, des visées, des attentes et peut-être bien que le temps m'est aussi compté, comme toujours, mais j'accepte au moins ici de rétrocéder, de revenir à ce que je croyais acquis, j'accepte de suspendre mes propres buts, mes propres urgences, pour laisser place non pas tant aux fins de l'élève, mais à son rythme propre, et aux incertitudes de ces rythmes.

Je dépasse également ou contiens ma frustration de constater que des choses qui devraient aller de soi ne vont pas du tout de soi, et que par conséquent il me faut rétrocéder, revenir en arrière, ralentir.

Qu'il faille une âme forte, cela tient aussi à ceci que justement nous acceptons de rétrocéder sur nos fins, non pas de les abandonner, mais bien de ralentir ou les suspendre un moment. La force ici est justement cette capacité à suspendre nos fins, à les mettre entre parenthèses pour un moment, en sachant pourtant, ou en tenant ferme sur le point que nous parviendrons à ces fins, quitte à faire des longs détours. La force est, en ce sens, patience, savoir "que le temps travaille pour nous", que souterrainement les choses se font, que nous en viendrons à ce que nous souhaitons. Ni retrait, ni forçage, mais, justement, patience et confiance dans le temps. Non pas une confiance aveugle, qui dit simplement que cela se fera, mais une confiance soutenue par les expériences acquises dans le passé qui nous permet souvent de deviner le signes des frayages qui se font.

La dévolution ainsi engage l'expérience pédagogique dans un certain temps. Et il faut hauteur d'âme, et force pour tenir dans ce temps.

Peut-être pourrions-nous ouvrir un peu les choses en disant que cette expérience du trotter nous conduit à ceci: peut-être que si moi je n'y arrive pas, tel autre pourra y arriver. On cède ici sur une certaine croyance selon 
laquelle notre langage, nos procédures, sont forcément bonnes; on accepte un partage des voies, sans pour autant le moins du monde renoncer aux siennes. Peut-être que là où je ne peux pas, un autre pourra réussir. Ce n'est pas seulement une formule d'irresponsabilité, une vague croyance, ou un vague espoir; c'est aussi l'expérience qu'il s'est avéré que là où je ne pouvais pas, d'autres ont pu réussir. Il faudrait penser cette expérience dans la richesse subjective qu'elle suppose, et en particulier dans la reconnaissance d'une limite personnelle, non accidentelle tel que je suis, cela ne m'est pas possible, tel que je suis, dans ce moment là, et selon une certaine situation, ou selon l'historique de cette situation, non de moi-même. Et la distinguer de l'aveu d'une impuissance.

Et bien sûr ici la déontologie n'est pas tant du côté de l'attention aux initiatives, mais plutôt du côté de la patience qu'il faut pour, peu à peu, réguler l'activité, la faire entrer dans des voies plus plastiques, moins chaotiques. Travail de la répétition, en tant qu'elle permet de s'assouplir.

Si la dévolution est attention aux initiatives, elle est aussi attention à la répétition elle-même, à l'effort de répétition, pour réguler, apaiser les muscles et les craintes. Je retrouve là les deux formes d'attention soulignées plus haut, les deux formes scolaires.

\section{Curiosité, créativité.}

On l'avertira, étant en compagnie, d'avoir les yeux partout; car je trouve que les premiers sièges sont communément saisis par les hommes moins capables, et que les grandeurs de fortune ne se trouvent guère mêlées à la suffisance.

J'ai vu, cependant que l'on s'entretenait, au haut bout d'une table, de la beauté d'une tapisserie ou du goût de la malvoisie, se perdre beaucoup de beaux traits à l'autre bout.

Il sondera la portée d'un chacun: un bouvier, un maçon, un passant; il faut tout mettre en besogne et emprunter chacun selon sa marchandise, car tout sert en ménage; la sottise même et faiblesse d'autrui lui sera instruction. ${ }^{8}$ 
Ici je m'appuie sur une autre image de Montaigne. La table d'hôte, l'enfant à une table d'hôte. Lattention est le plus souvent orientée vers le "haut bout de la table", et certes, ce n'est pas sans raison. Ce haut-bout renvoie aux personnes de valeurs. Et donc plus largement aux choses de valeurs. Et au fond, c'est là quelque chose d'important pour l'éducation et l'école. On y transmet, comme on dit, ce qu'il y a de plus hauts et de plus noble dans notre patrimoine culturel. Montaigne parlera de cela et dira que les meilleurs objets de la transmission sont ceux par lesquels déjà beaucoup sont passés. Et, si beaucoup y sont passés, cela augure du fait que nous aussi nous pourrons y trouver notre bien.

Mais Montaigne dit aussi autre chose: et vise la capacité à s'intéresser des choses qui sont, pour les gens attablés, sans valeur. Dire cela, c'est dire que l'attention de l'élève peut être elle-même créatrice de valeur; qu'il est autrement dit souhaitable et possible que l'enfant porte intérêt à "des riens", et, par là, crée pour lui-même de la valeur. Et s'intéresser à des riens, c'est cesser de s'intéresser à ce qui intéresse tout le monde, de forcément s'intéresser à ce qui intéresse tout le monde, de la même façon que tout le monde. Je crois qu'il y a là une source, sinon la source même de toute créativité, au sens où celle-ci commence dans cette capacité de trouver de la valeur sans écriteau ou parole nous disant que c'est là où nous devrions la chercher.

Il ne s'agit pas d'opposer de façon morale ces deux types d'attention, mais de dire qu'elles ont besoin l'une de l'autre, qu'elle doivent être prises en compte l'une et l'autre, et pour ce qui concerne la seconde, qu'elle doit être travaillée. Il ne va pas de soi de s'intéresser à des riens. Cela risque justement de n'intéresser personne! Et pourtant, cela a requis notre attention et notre intérêt. Il faut alors pouvoir le développer sans assurance.

Dévoluer le jugement, c'est ainsi solliciter et permettre la capacité à trouver intérêt à des riens, à signifier que telle chose, négligée, est en fait bien suggestive. Je retrouve là la notion d'attention que j'avais abordée plus haut. Lattention n'est pas seulement attention à ce qui vient, à ce qui peut venir, elle est aussi une capacité à se détourner de ce que nous nommons réponses toutes faites, ou de ce que nous savons comme par avance que cela vaut. Elle neutralise la question de la valeur, et par cela même l'ouvre, vers ce qui ne semble pas en avoir, et pourtant se trouve là. Le médecin, lorsqu'il fait un diagnostic, ne fait-il pas cela? A la fois attentif aux signes habituels, "attendus" mais faisant jouer une certaine attention diffuse autour, une attention flottante comme dit Freud pour un autre contexte? Et cela à côté de la connaissance de ce qui a intérêt, de ce qui aura intéressé et de ce qui pourrait aussi m'intéresser à ma manière. 


\section{Les livres, le rapport aux livres}

Enfin, je m'attarderai sur un autre passage de Montaigne qui me semble de nouveau bien instructif.

Après qu'on lui aura dit ce qui sert à le faire plus sage et meilleur, on l'entretiendra que ce n'est que logique, physique, géométrie, rhétorique; et la science qu'il choisira, ayant le jugement déjà formé, il en viendra bientôt à bout. Sa leçon se fera tantôt par devis (conversation) tantôt par livre; tantôt son gouverneur lui fournira de l'auteur même, propre à cette fin de son institution; tantôt il lui en donnera la moelle et la substance toute mâchée. Et si de soi-même il n'est pas assez familier des livres pour y trouver tant des beaux discours qui y sont, pour l'effet de son dessein, on lui pourra joindre quelques hommes de lettres, qui à chaque besoin fournira les munitions qu'il faudra, pour les distribuer et dispenser à son nourrisson. ${ }^{9}$

On retrouve là la thématique de l'alternance que nous n'avons pas cessé de croiser("tantôt... tantôt"). Comme on le voit elle s'élargit selon des formes tout à fait suggestives.

Lattention désormais s'ouvre aux savoirs et aux livres. Elle le fait toutefois en alternant une approche orale, synthétique, où il s'agit de dire l'essentiel et l'important d'un texte donné, et une approche que l'on peut dire plus studieuse et qui se penche sur les textes eux-mêmes. Montaigne semble ainsi présupposer que le mode d'approche d'un texte quelconque du savoir est en fait double, ou encore qu'il correspond à deux modes d'attention de l'esprit, qui ne peuvent se recouvrir. D'un côté la lettre et son étude, de l'autre ce que l'on peut en dire, comme s'il y avait un saut entre les deux, ou pas de solution de continuité. Il y a "ce que dit le texte", et il y a "ce que nous pouvons en dire, ou en extraire, nous-mêmes", pourrions-nous dire, ce par quoi il nous enseigne quelque chose.La lecture, comme l'apprendre, comme l'apprentissage ne sont possibles que sur le fond d'une telle alternance. Lire, c'est, à un moment ou un autre, se risquer, et proposer une certaine lecture, puis comme on sait revenir. C'est bien ce que la tradition herméneutique cherchera à analyser plus tard, et particulièrement H. G. Gadamer. Mais tout l'intérêt de cette citation est de rapporter cette différence à la différence de

9 MONTAIGNE, Les Essais, I,26, 1990, p.123. 
l'écrit et de l'oral. Sans doute est-ce bien ici le précepteur qui fait jouer alternativement ces deux rapports, mais, procédant lui-même ainsi, il en suggère la possibilité à son élève et l'ouvre par là à cette différence.

Il faudrait bien sûr examiner en quoi cette différence dit quelque chose d'essentiel à l'esprit et si également les théories cognitivistes contemporaines nous en disent quelque chose. Mais déjà elle nous semble importante en tant qu'elle instaure un certain pluralisme de la relation tant à soi-même qu'à autrui et aux savoirs. Il y a là la constitution de ce que l'on peut nommer une mobilité psychique: ne pas coller aux textes du savoir, ne pas non plus ignorer ou survoler ces textes, mais précisément alterner. Revenir à soi, faire quelque chose du texte, et revenir au texte, aux savoirs eux-mêmes, sans présupposer de fins à ce mouvement. Montaigne, très régulièrement, fera pour lui témoignages de ces allers et retours.

C'est enfin sur le fond de cette alternance que Montaigne est conduit à introduire d'autres protagonistes de l'éducation, à savoir des spécialistes de telle ou telle discipline, pour aider le précepteur. Là encore, on peut faire l'hypothèse qu'il s'agirait d'une familiarisation à d'autres modes de discours, autant qu'à d'autres modes du rapport aux savoirs. C'est bien un nouvel étage du pluralisme, qui implique cette fois-ci d'autres protagonistes.

Comme on le sait, ce pluralisme intégra également la tradition philosophique dont Montaigne dit ceci :

Ce grand monde, que les uns multiplient encore comme espèces sous un genre, c'est le miroir où il nous faut regarder pour nous connaître de bons biais. En somme, je veux que ce soit le livre de mon écolier. Tant d'humeurs, de sectes, de jugements, d'opinions, de lois et de coutumes nous apprennent à juger sainement des nôtres, et apprennent notre jugement à reconnaître son imperfection et sa naturelle faiblesse: ce qui n'est pas un léger apprentissage. (...) On a grand tort de la peindre (la philosophie) inaccessible aux enfants, et d'un visage renfrogné, sourcilleux et terrible. Qui me l'a masqué de ce faux visage, pâle et hideux? Il n'est rien plus gai, plus gaillard, plus enjoué, et à peu que je ne dise folâtre. Elle ne prêche que fête et bon temps. ${ }^{10}$

C'est ainsi le dernier étage d'un certain pluralisme que vise Montaigne, et que les philosophies démontrent semble-t-il dans leur existence même, et

10 MONTAIGNE, Les Essais, I,26, 1990, p.121 et 123 
dans le type d'homme que sont les philosophes. J'ai cherché à dessiner, en suivant Montaigne, les conditions concrètes et, semble-t-il, primaires, d'une telle éducation à ce monde pluriel.

\section{Conclusion}

Je me serai donc appuyé sur ce texte de Montaigne pour construire un premier temps de ma réflexion sur ces questions de didactique et/ou de pédagogie. Et cela en mobilisant les philosophes eux-mêmes, si l'on veut bien compter Montaigne parmi les philosophes. Certes, autorité n'est pas raison, même si, souvent, elle peut contribuer à nous éclairer et à faire naître des attentions un peu nouvelles. C'est bien la forme d'une certaine alternance qui m’a semblé pouvoir retenir ici notre attention. C'est en elle que se joue ce que Montaigne nomme la formation du jugement, comme mouvement et articulation de deux spontanéités et des reprises qu'elles se font mutuellement. J'ai cherché à montrer différents aspects de ce jeu, en soulignant en particulier les points où il s'articule avec un certain jeu avec le monde. Et dans cette perspective j'ai bien croisé des thèmes qui, pour être peu originaux, n'en sont pas moins importants.

Mais je poursuivrai cette enquête dans un article ultérieur consacré à Alain.

\section{Références bibliographiques}

BOUVERESSE,Jacques:Le mythe de lintériorité.Paris : Ed. de Minuit, 1987 ?

BRUNER, Jérôme: Savoir dire, savoir faire. Paris : PUF, 2011

MONTAIGNE, Michel : Les Essais, I,26. Paris: Arléa, 1990 